\title{
Research and Implementation of the UDS Diagnostic System
}

\author{
Lingfeng $\mathrm{Xie}^{1, \mathrm{a}}$, Feng Luo ${ }^{2, \mathrm{~b}}$ \\ ${ }^{1,2}$ Clean Energy Automotive Engineering Center of Automotive Studies, Tongji University, China \\ a87xielingfeng@tongji.edu.cn, bluo_feng@tongji.edu.cn
}

Keywords: UDS protocol; CAN to Wi-Fi/Bluetooth; host computer; handheld terminal equipment.

\begin{abstract}
As an automotive diagnostic technology, the UDS (unified diagnostic services) protocol has been more and more widely applied. In this paper, a diagnosis system based on UDS is developed and tested. According to the UDS protocol, the software is designed and groups of diagnostic services are implemented. Besides, the host computer is designed so that the results of diagnosis can be parsed and displayed clearly. The host computer is divided into two types: one is implemented on PCs, which the communication mode is based on $C A N$ bus; the other one is implemented on the handheld terminal equipment, the Android pad, which a $C A N$ to $\mathrm{Wi}$-Fi/Bluetooth hardware module needs to be designed so that the $C A N$ frame can be translated from the microprocessor to the handheld terminal equipment. Finally, in order to verify the diagnostic system, we carry out some test cases and draw the conclusions that the diagnostic system is feasible.
\end{abstract}

\section{Introduction}

The $C A N$ bus is the main standard in the current automobile network system of high speed. It supports the speed of $1 \mathrm{M} / \mathrm{s}$ and distributed real time control with a high level of security and relatively low cost [1]. Nowadays, most of the vehicles use the CAN bus as their mode of communication.

The diagnostic technology in automotive refers to that acquiring the internal state information, finding the location of diagnosis and solving it in time without dismantling vehicles. With the widespread of the CAN bus, the ISO distributes a series of protocols to realize the standardization of diagnosis based on $C A N$ bus. So the UDS (Unified diagnostic services) protocol was born [2]. With the help of this protocol, the efficiency of diagnose has been improved [3].

The UDS protocol is also called the ISO 14229-1 protocol [3], which has been established to define the common requirements and services for diagnostic systems in application layer. And the ISO 15765-2 [4] defines the transport protocol and network layer services. The UDS protocol allows that the diagnostic tester called client to build a communication with the diagnostic target ECU called server by $C A N$ bus. The client mainly reads/writes the data from/to the flash of controllers, re-programming of ECUs, etc. The client translates the requests of diagnostic services defined in UDS and the sever carries out these diagnostic services. After carrying out these requests successfully, the server needs to return a possible message to the client, otherwise returning a negative message. The diagnosis services mainly conclude that reading DTC (Diagnostic Trouble Code), clearing DTC, reading and writing DID (DataByIdentifier) and 25 diagnostic services in total. Every service occupies their own ID named SID to differ from other services. And there are some sub-functions in most number of services to realize different functions.

\section{System Architecture}

In this paper, the diagnostic software based on UDS is developed [6,7]. At the same time, two host computers, which one is on $P C$ and the other one is on handheld terminal equipment Android pad are also designed to test the diagnostic software. Via this diagnostic system and host computer, the diagnostic function of ECU can be implemented and tested.

The figure 1 refers to the diagnostic system with the host computer based on the $P C$. The system includes 4 parts: the ECU with diagnostic software, CAN bus, SuperCAN and host computer. The 
SuperCAN helps the PC convert $C A N$ frame data and serial data, so the communication between host computer and $E C U$ can be realized via $C A N$ bus.

The figure 2 refers to the diagnostic system with the host computer based on the hand terminal equipment. The system also includes 4 parts: the ECU with diagnostic software, Wi-Fi/Bluetooth model, $C A N$ bus and host computer. The Wi-Fi/Bluetooth model helps the hand terminal equipment convert CAN frame data and Wi-Fi/Bluetooth data. The advantage of this system is the communication convenient and can be realized via wireless.

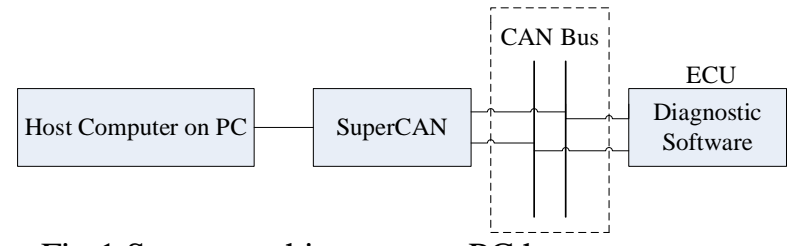

Fig 1.System architecture on PC host computer

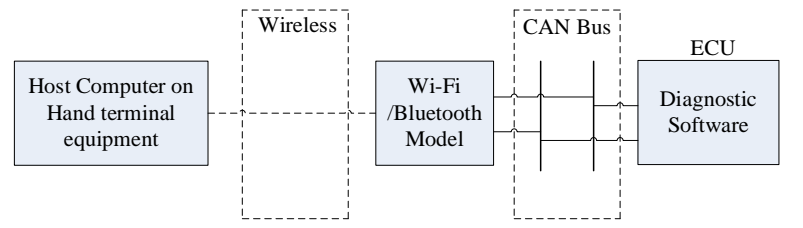

Fig 2.System architecture on hand terminal equipment

\section{Diagnostic Software Design}

Figure 3 refers to the software architecture. The software runs in a loop. Once a diagnostic request message is translated from the client, the server will catch the CAN frame and translate it to the network layer. The network layer will unpack and parse the $C A N$ message, judging whether the message is single or multi frames, and whether the message needs to return a flow control frame. Then the rest messages will be translated to the application layer. The application layer of the server mainly unpacks and acquires the SID and sub-function ID to implement the specified diagnostic service. Finally, the application layer will pack and translate the state information to the network layer. The network layer will determinate to return single or multi $C A N$ frames, then returning the message.

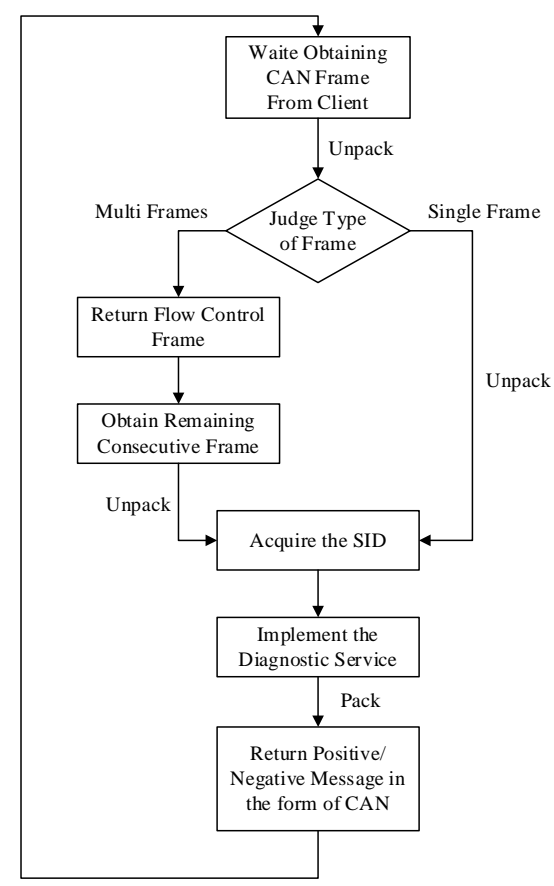

Fig 3.Software architecture

Figure 4 shows the process of message transmitting. First and foremost, in the lowest layer, the physical layer, client and server are communicated via CAN bus. So the CAN model should be initialized, and some attributes are also set, such as baud rate. When receiving a $C A N$ frame, the server will come into an interrupt and check the $I D$ of the message whether is related to the diagnosis. The server will save the $C A N$ message that is related to the diagnosis in its cache, and wait to be handled by the network layer. After implementing above operation, system exits the interrupt and waits for the next $C A N$ frame. 
In the network layer, an interface function should be defined so that the data can be translated between the network layer and application layer. The interface function will be called when the message is saved in the cache. Via the interface function, the software can realize the packing or unpacking function of the message. When translating message, the network layer determinates the message to translate in the form of the single or multi frames according to the length of the message in interface function. When receiving message, the network layer will unpack the $C A N$ frame according to the protocol control information so that the application layer can understand which type of the CAN frame is belong to and which diagnostic services should be implemented.

The top layer, application layer, only handles the message from the network layer rather than need to consider the way of translation.

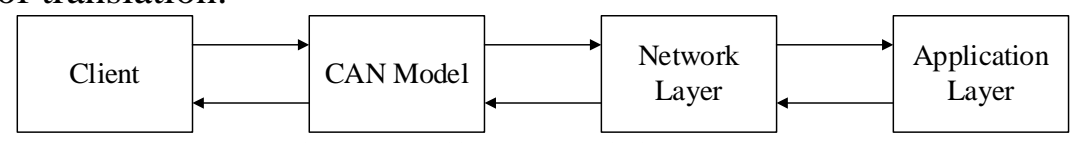

Fig 4. Detailed transmitting process

There are two forms of the transportation, which is judged by the network layer:

A. When receiving an unsegment message from client, the mode of transportation see figure 5.

B. When receiving a segment message from client, the mode of transportation see figure 6 .

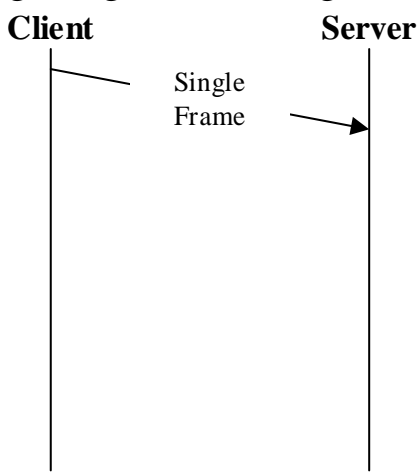

Fig 5.Receiving an unsegment message

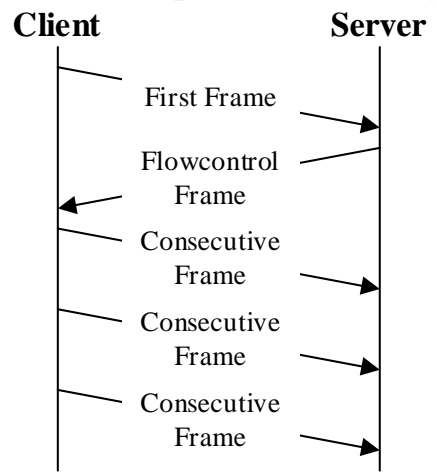

Fig 6.Receiving an segment message

\section{Host Computer Design}

After designing the software, the host computers are also developed. The host computer is designed according to the UDS protocol. Its function mainly concludes translating and parsing the diagnostic $C A N$ frame return from the server to distinguish that which the diagnostic services are implemented and acquire some useful information. In this paper, two types of host computers are designed: one is based on the PC, the other one is based on the handheld terminate equipment.

A. The host computer based on $P C$

The host computer on the PC is developed on the basis of the VisualStudio2012. It can run normally on Windows $X P / 7$ operating system. The design ideas are divided in below 4 steps:

First, the $P C$ is accessed to the $C A N$ bus by using the SuperCAN because the $P C$ can't receive the $C A N$ frame directly and the tool SuperCAN can translate the $C A N$ frame into the serial data that $P C$ can understand. Calling the library functions of the SuperCAN in this host computer so that the communication has been built.

Second, realizing the function of translation and reception of the CAN frame according to the $C A N$ standard so that the client can filter the $C A N$ frames from the serves and translate the diagnostic $C A N$ frames to the target server via inputting the $I D$.

Third, realizing the diagnostic services defined in UDS protocol. The content and analysis of different diagnostic services are different. It is important to judge whether the frame is single or multi.

Finally, designing the operation interface, planning the layout of every component and realizing the trigger conditions of all diagnostic services to achieve a better human-computer interaction. 
The operation interface are as figure 7. First step of operation is building communication, then inputting the $I D$ of the server and choosing the specific diagnostic service, and fill in the relevant data information. After that, select the "Execute Diagnose" button, then the host computer will display the results of this diagnosis on the lower right.

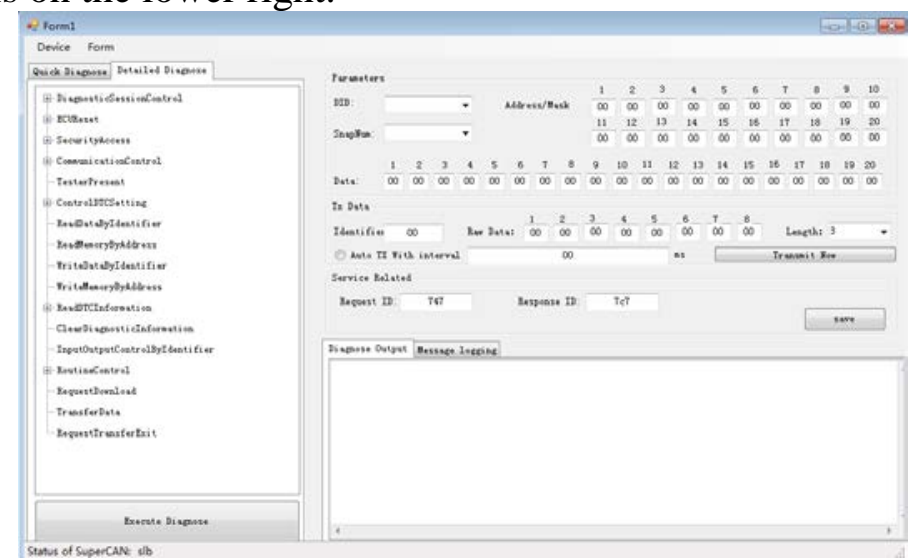

Fig 7.Operation interface on host computer based on PC

B. The host computer based on handheld terminate equipment

The host computer based on the handheld terminate equipment is developed on the basis of the android pad by Android Studio, because the Android OS is the most popular OS nowadays and the android pad is portable and easy to use. The operation interface on the android pad refers to figure 8 .

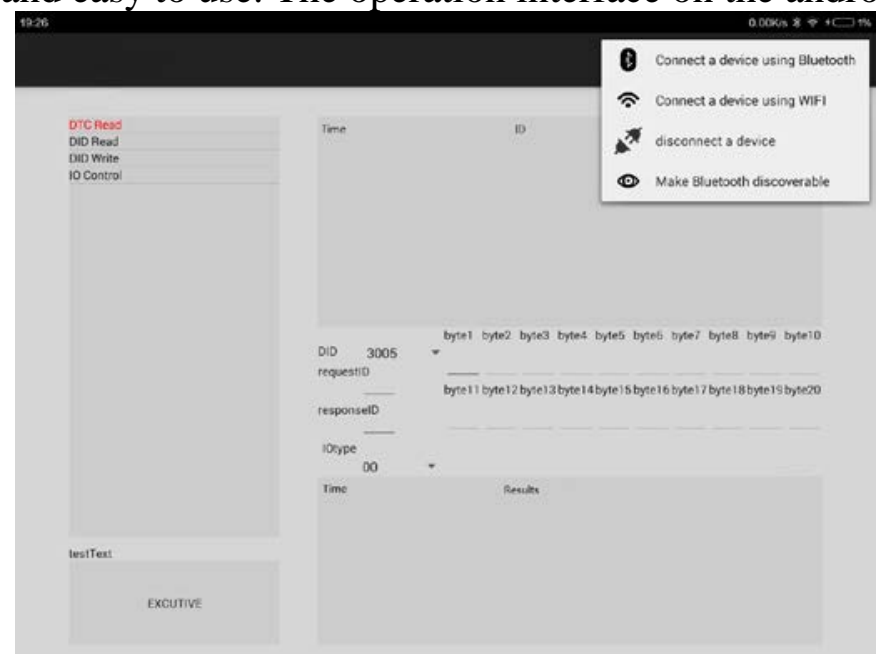

Fig 8. Operation interface on host computer on handheld terminal equipment

Because the android pad can't receive the CAN frames directly and it supports the wireless communication of Wi-Fi and Bluetooth. Designing a hardware module that converting CAN to $\mathrm{Wi}-\mathrm{Fi} /$ Bluetooth is a primary task. With the help of this module, the message can convert between the CAN data and Wi-Fi/Bluetooth data.

The architecture of this host computer is mainly divided into 3 layers: the bottom layer is used to configure the Wi-Fi/Bluetooth hardware modular and build connection so that the reception and translation of $C A N$ frames can be achieved. The second layer is network layer that is used to pack and unpack the $C A N$ frame. The top bottom is used to interactive with the users.

The workflow of the host computer based on android pad are basically the same as that on the PCs.

\section{System Testing}

The testing systems shown in figure 9 is divided into two examples, one is based on the PCs and another one is based on the handheld terminal equipment. 


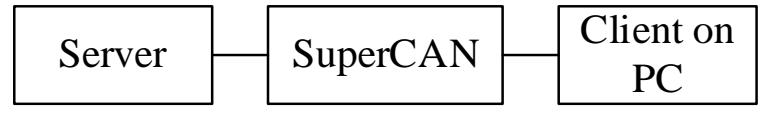

(a).Testing system on PC

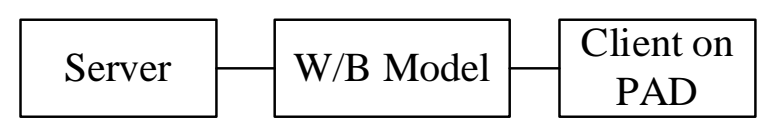

(b). Testing system on handheld terminal equipment

Fig 9. Testing system

Setting one of the most common diagnostic service, reportDTCByStatusMask as an example, whose SID and the code of the sub-system are 0x19 and 0x02 respectively. Its function is used to read the current DTC in the server ECU. After building the testing system, considering that the situation of translating the multi CAN frames, making the server produce multi troubles, whose DTCs are 0x10822, 0x10823, 0x10824 and 0xD0825. The displays are shown as figure 10:

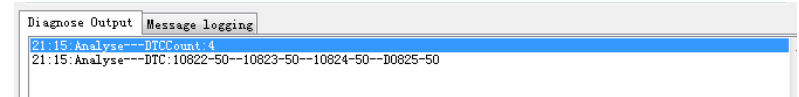

(a). Host computer on PC

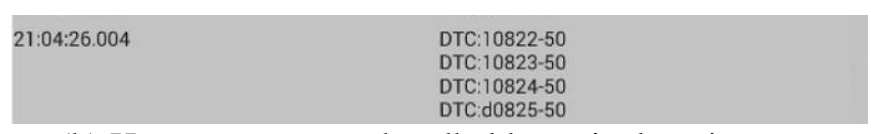

(b).Host computer on handheld terminal equipment

Fig 10. Display of the parsing return when multi trouble

From the figure 10, multi DTCs can also be displayed correctly both in the 2 host computers. After multiple groups of tests, the two host computers can display the right results.

Every diagnostic service defined in the UDS protocol has been tested with the two host computers. And all the services has passed the test.

\section{Summary}

As a standard for automobile diagnostic protocol, the UDS protocol based on the CAN bus can judge where and which diagnoses take place more efficiently with the improving number of the $E C U$ in the vehicle. This paper develops the software on the basis of the UDS protocol with two types of host computers to verify its feasibility. Finally, the diagnostic software system passes all the tests and proves its correctness and consistency with the UDS protocol.

\section{References}

[1] BOSCH CAN Specification 2.0.

[2] Junkui Huang, Jinrui Nan, Zhi Chai, and Cheng Lin. Analysis of UDS Diagnostic Service Applied on Vehicle ECU [J]. Applied Mechanics and Materials (2013).

[3] Miller Jason, Thomas Tim, Waldeck, and Bill. Challenges and Benefits to Adopting a Worldwide Diagnostic Protocol Specification[C]. SAE World Congress (2004).

[4] ISO 14229-1:2013 Road vehicles - Unified Diagnostic Services (UDS) - Part1: Specification and Requirements.

[5] ISO 15765-2:2011 Road vehicles - Communication over Controller Area Network (DoCAN) Part2: Transport Protocol and Network Layer Services.

[6] Wajape, Mahesh, Elamana, and Nithin. Study of ISO 14229-1 and ISO 15765-3 and Implementation in EMS ECU for EEPROM for UDS Application [C]. 2014 IEEE International Conference on Vehicular Electronics and Safety (ICVES) (2014).

[7] Song Yan, Wang Tianran, Xu Aidong, Wang Kai, and Yang Zhijia. CAN based Unified Customizable Diagnostic Measure Research and Realization [C]. UKACC International Conference on Control (2012). 